\title{
OCUPACIÓN HUMANA PLEISTOCÉNICA EN EL DESIERTO DE ATACAMA: PRIMEROS RESULTADOS DE LA APLICACIÓN DE UN MODELO PREDICTIVO DE INVESTIGACIÓN INTERDISCIPLINARIA*
}

\author{
PLEISTOCENE HUMAN OCCUPATION IN THE ATACAMA DESERT: \\ FIRST RESULTS FROM THE APPLICATION OF AN INTERDISCIPLINARY \\ PREDICTIVE RESEARCH MODEL
}

\author{
Calogero M. Santoro ${ }^{1,2}$, Paula C. Ugalde ${ }^{2 *}$, Claudio Latorre $^{3,4,7}$ Carolina Salas $^{5}$, \\ Daniela Osorio ${ }^{2}$, Donald Jackson ${ }^{6}$ y Eugenia Gayó ${ }^{7}$
}

\begin{abstract}
Sudamérica cuenta con evidencias de ocupación humana desde los 14.600 años calibrados a.p. (14,6 ka). Sin embargo, en el desierto de Atacama existe una notoria ausencia de ocupaciones pre-11,5 ka entre las latitudes $17^{\circ}$ a $21^{\circ} \mathrm{S}$ (Atacama Norte). Esto debido, en parte, a que se ha desestimado como territorio atractivo para poblaciones de la transición Pleistoceno-Holoceno, dadas sus condiciones actuales de extrema aridez. Sobre la base de ciertos datos paleoecológicos generales, que sugerían condiciones de mayor disponibilidad de agua en la vertiente occidental de los Andes desde los 17 a 9,5 ka, se realizaron búsquedas sistemáticas de paleomadrigueras de roedores y paleohumedales en ambientes de precordillera y quebradas (1.000 a $3.000 \mathrm{msm}$ ). Esto permitió identificar hábitat específicos que presentaran condiciones favorables para el asentamiento humano temprano y de esta manera buscar sitios arqueológicos en forma dirigida. El modelo metodológico interdisciplinario de carácter predictivo, que se resume en este artículo, permitió identificar un conjunto de sitios entre los que destaca Quebrada Maní 12, donde se constataron las primeras evidencias de ocupaciones pleistocénicas tardías ( 11,9 a 12,7 ka) en el desierto de Atacama Norte.
\end{abstract}

Palabras claves: transición Pleistoceno-Holoceno, poblamientos tempranos, paleoambiente, Desierto de Atacama.

In South America, evidence of human occupation dates back to 14,600 calibrated years BP (14.6 ka). Yet, important areas such as the Atacama Desert, between latitude $17^{\circ}$ to $21^{\circ} \mathrm{S}$ (northern Atacama), lack occupations older than 11,5 ka. Current hyperarid conditions in the Atacama have dissuaded many researchers from considering this region as a possible territory for Pleistocene-Holocene peoples. Paleoecological data, however, have suggested increased availability of water along the western slope of the Andes from 17.5-9.5 ka. Thus, we systematically searched for rodent middens and paleowetlands in the large canyons of the Andean Precordillera as well as the interfluves (1,000-3,000 masl). As a result, we identified specific habitats favorable for early human settling. This interdisciplinary and predictive methodological model, summarized in this paper, allowed us to identify several sites. Among these, Quebrada Maní 12 is the first Pleistocene-Holocene human occupation ( 11.9 a 12.7 ka) known from the northern Atacama.

Key words: Pleistocene-Holocene transition, early peopling, paleoenvironment, Atacama Desert.

* Un texto preliminar de este artículo fue presentado en el 53 Congreso Internacional de Americanistas, Simposio "El período Arcaico en los Andes Sur Centrales: Tradiciones culturales e innovaciones tecnológicas”, Ciudad de México, 19 al 24 de julio de 2009. Este manuscrito fue evaluado por investigadores externos y editado por Luis Flores Blanco y Mark S. Aldenderfer, en su calidad de editores invitados de la Revista.

1 Instituto de Alta Investigación, Departamento de Antropología, Universidad de Tarapacá, Casilla 6-D, Arica, Chile. calogero_santoro@yahoo.com

2 Centro de Investigaciones del Hombre en el Desierto, Av. General Velásquez 1775, Arica, Chile. daniosorio@gmail.com * Autor correspondiente: pugalde@ cihde.cl

3 Centro de Estudios Avanzados en Ecología y Biodiversidad, Pontificia Universidad Católica de Chile, Casilla 114-D, Santiago, Chile. clatorre@bio.puc.cl

4 Instituto de Ecología y Biodiversidad Universidad de Chile, Las Palmeras 3425, Nuñoa, Santiago, Chile.

5 Programa Magíster en Antropología, Universidad Católica del Norte, San Pedro de Atacama, Chile. carito_s@ yahoo.com

6 Departamento de Antropología, Universidad de Chile, Ignacio Carrera Pinto 1045, Santiago, Chile. djackson@uchile.com

7 Departamento de Ecología, Pontificia Universidad Católica de Chile, Casilla 114-D, Santiago, Chile. egayoher@bio.puc.cl 


\section{Problema y el Modelo Predictivo}

A partir de la finalización del Último Máximo Glacial (ca. 17 ka), la región occidental del continente sudamericano comenzó a experimentar mayores temperaturas y un aumento sostenido de las precipitaciones en las regiones tropicales (Placzek et al. 2009). Esto, por efecto de una mayor disponibilidad de agua proveniente de los Andes Centrales en su vertiente occidental (Placzek et al. 2009; Quade et al. 2008). Coincidentemente, Sudamérica comienza a colonizarse a partir de esa época, de tal manera que hacia los 13 ka se registran evidencias de ocupación humana en el extremo sur del continente (Patagonia y Tierra del Fuego), el borde norte y noreste (Colombia, Venezuela y Brasil) y especialmente a lo largo de la vertiente occidental de los Andes (Dillehay 2000). En esta última región es donde se ha registrado la mayor densidad y diversidad de sitios tempranos. Todos ellos antecedidos, sin embargo, por Monte Verde, con una datación promedio de 14,6 ka (Dillehay 1989, 1997, 2000; Jackson et al. 2007; Kelly 2003: fig. 1; Lynch 1980:fig. 1; Rothhammer y Dillehay 2009: fig. 1).

Resultados recientes de varios grupos de estudios paleoambientales independientes concuerdan en que el Desierto de Atacama $\left(\sim 17^{\circ}\right.$ a $\left.27^{\circ} \mathrm{S}\right)$ gozó de condiciones menos áridas que las actuales entre $\sim 17$ a 9,5 ka (Betancourt et al. 2000; Gayó et al. 2010; Grosjean et al. 2005a; Latorre et al. 2006; Latorre et al. 2005; Moreno et al. 2007; Nester et al. 2007; Rech 2001; Rech et al. 2001, 2002). Se estima que el sistema hidrográfico del Atacama, que depende de las precipitaciones en la vertiente occidental de los Andes, tuvo un aumento de hasta cerca del $100 \%$ en relación a los promedios actuales que van entre $300 \mathrm{~mm}$ anuales en el Atacama Norte (ca. $\left.17-21^{\circ} \mathrm{S}\right)$ y $150 \mathrm{~mm}$ en el Atacama Sur ( 25-27 S). Esto tuvo como consecuencia: (a) una mayor recarga de acuíferos y cauces superficiales desde los Andes Centrales hasta el Pacífico y el afloramiento de vertientes a los pies de los Andes, (b) aumento de precipitaciones en territorios bajo los $3.000 \mathrm{~m}$, altura que actualmente muestra un déficit hídrico extremo, (c) un descenso de especies altiplánicas y de la puna hacia el piso pre-puneño a $2.000 \mathrm{~m}$, y (d) florecimiento de bosques en parches o galería, en zonas bajas y actualmente secas en márgenes de la Pampa del Tamarugal (cuenca endorreica ubicada entre $19^{\circ} 17^{\prime}$ y $21^{\circ} 30^{\prime} \mathrm{S}$, a una altura promedio de $1.000 \mathrm{msm}$ ).
Varios estudios arqueológicos se han destinado a la búsqueda de evidencias tempranas en el norte de Chile y sur de Perú (Dauelsberg 1983; de Souza 2004; deFrance y Umire 2004; Grosjean et al. 2005b; Lanning 1967, 1973; Le Paige 1965, 1970; Lynch 1986; Núñez 1975; Núñez et al. 2002; Salazar et al. 2011; Sandweiss et al. 1998; Schiappacasse y Niemeyer 1984). El registro de una gran cantidad de sitios, especialmente a lo largo de la costa y las tierras altas (>3.000 msm), y de más de 800 dataciones radiocarbónicas demuestran la intensidad con que se ha explorado el Desierto de Atacama (Williams et al. 2008). Sin embargo, sólo se han localizado y datado siete sitios previos a 11,5 ka. Los primeros seis ubicados en el Atacama Central (ca. 22-24 S): Tuina-5, Tuina-1, Tulán-109, San Lorenzo (Núñez et al. 2002), Salar de Punta Negra-1 (Núñez et al. 2005) y Alero el Pescador (de Souza 2004). El último, San Ramón 15 (Salazar et al. 2011), está localizado en el Atacama Sur $\left(25-27^{\circ} \mathrm{S}\right)$. En el extremo sur del desierto costero peruano se ubican dos áreas arqueológicas, datadas igualmente hacia fines del Pleistoceno: Tacahuay (deFrance y Umire 2004; Keefer et al. 1998) y varias localidades en la quebrada Jaguay (Sandweiss et al. 1998).

El escaso número de evidencias tempranas se debería, en parte, a que dadas las condiciones actuales de extrema aridez de la franja altitudinal entre los 1.000 y $3.000 \mathrm{msm}$ el Desierto de Atacama ha sido subestimado como territorio atractivo para poblaciones del Pleistoceno-Holoceno (Santoro y Latorre 2009). Por esta razón, raramente ha sido explorado arqueológicamente con el propósito específico de ubicar sitios de dicha época. Esto es particularmente cierto entre los $17^{\circ}$ a $21^{\circ}$ latitud sur (Atacama Norte), donde existe una notoria ausencia de sitios anteriores a 11,5 ka. Estimamos que este vacío no refleja el modelo de poblamiento del "salto de la rana" de Anderson y Gillam (2000), sino la carencia de diseños de investigación interdisciplinaria que consideren factores climáticos, geomorfológicos, hidrológicos y culturales, destinados específicamente a encontrar sitios tempranos.

Con estos antecedentes, definimos a modo de hipótesis que (1) La zona intermedia entre la Cordillera de la Costa y la precordillera andina (2.000-3.000 msm) presentó condiciones ambientales favorables en cuanto a clima y recursos bióticos para la ocupación humana durante la transición Pleistoceno-Holoceno (17 a 9,5 ka) (Betancourt et al. 2000; Latorre et al. 2006; Latorre et al. 2005; 
Rech 2001; Rech et al. 2002). En el transcurso de este estudio descubrimos que las condiciones climáticas favorables señaladas se extendieron hasta $\sim 1.000 \mathrm{msm}, \mathrm{y}$ (2) $\mathrm{Si}$ este supuesto era correcto, propusimos consecuentemente que este espacio no habría quedado exento de la temprana historia humana del continente, y que evidencias del paso de estas primeras poblaciones, desde el sur del Perú hacia el Atacama Central, deberían encontrarse en este territorio intermedio.

Estas hipótesis generaron un modelo predictivo interdisciplinario, que integra datos paleoecológicos y geológicos para localizar y caracterizar ambientes atractivos para la ocupación humana durante el período de estudio (Santoro y Latorre 2009). A partir de ello se realizaron prospecciones intensivas y sistemáticas para la búsqueda, identificación y datación de sitios arqueológicos de data temprana, desde los 18 hasta los $25^{\circ} \mathrm{S}$ (Valle de Lluta hasta la quebrada Agua de Cascabeles al interior de Taltal; Figura 1).

\section{Metodología}

Nuestra estrategia paleoambiental se sustentó en cuatro líneas de evidencias: (1) registros lacustres que indican cambios en la humedad disponible (Moreno et al. 2009; Placzek et al. 2009), (2) registros de paleohumedales y paleovertientes que indican cambios en la altura y posición de las napas freáticas (Quade et al. 2008; Rech 2001; Rech et al. 2002), (3) paleomadrigueras de roedores que indican cambios vegetacionales y variaciones en las precipitaciones locales (Latorre et al. 2006) y (4) terrazas fluviales con remanentes fosilizados de vegetación ribereña (Nester et al. 2007).

El estudio preexistente de paleovertientes, lugares donde se habrían concentrado los recursos bióticos (Rech 2001; Rech et al. 2002), fue un punto de partida clave para el diseño de esta investigación y para dirigir las primeras búsquedas arqueológicas. A esto se sumó la identificación de nuevos enclaves con paleovertientes.

Además, reconstrucciones paleoambientales regionales y locales se obtuvieron del análisis de paleomadrigueras de roedores. Éstas, verdaderos archivos de cambio biológico y de información paleoambiental, corresponden a acumulaciones de restos macro y microorgánicos (plantas, cutículas, polen, insectos y huesos) (Betancourt et al. 2000; Betancourt y Saavedra 2002; Betancourt et al. 1990). Las madrigueras mejor conservadas se ubican en formaciones de rocas de calizas, areniscas, brechas y rocas volcánicas (especialmente ignimbritas, las que típicamente caracterizan el paisaje geológico andino y por lo tanto factibles de ser encontradas junto a depósitos geológicos de paleovertientes). Dado que el área potencial de forrajeo de los roedores abarca generalmente $<100$ $\mathrm{m}$, se realiza un muestreo de la vegetación local en un área de $500 \mathrm{~m}$ alrededor de la madriguera. Los depósitos de roedores, que presentan normalmente la consistencia de un ladrillo de adobe, se extraen utilizando martillos y cinceles, poniendo especial cuidado a cambios microestratigráficos que pueden reflejar períodos de abandono y reocupación de la madriguera (mayor información en Betancourt y Saavedra 2002).

Sobre la base del estudio de estos archivos paleoambientales, se ha logrado establecer un panorama general de las variaciones espaciales y temporales de las condiciones medioambientales en el Desierto de Atacama (Betancourt et al. 2000; Holmgren et al. 2001; Holmgren et al. 2008; Latorre et al. 2005; Latorre et al. 2002; Latorre et al. 2003). En términos específicos, permitió definir localidades puntuales donde se aplicaron las tácticas arqueológicas.

La estrategia arqueológica se articuló a partir de: (1) exploraciones arqueológicas intensivas en los hábitats definidos a través de los datos paleoambientales específicos previos y obtenidos por el proyecto, con miras a identificar sitios abiertos o en refugios rocosos, (2) sondeos con barreno, calicatas y excavaciones de mayor amplitud para determinar potencialidades estratigráficas, presencia de artefactos y materiales para datación. En todos los casos se recogió la totalidad del sedimento grueso, harneado con tamices de 2 a $3 \mathrm{~mm}$ de ancho, para su posterior análisis en laboratorio (p.ej., identificación de microartefactos). Del sedimento fino $(<2 \mathrm{~mm})$, recogido bajo el tamiz, se obtuvieron muestras de cada estrato (ca. 1 litro) para análisis químicos y arqueobotánicos (i.e. flotación), (3) recolecciones superficiales totales de cuadrantes con mayor densidad de artefactos o con la presencia de instrumentos tipológicamente tempranos, con miras a obtener conjuntos completos de materiales, (4) análisis macrobotánicos, para obtener una línea independiente de datos acerca de las condiciones ambientales locales, como también sobre el uso y consumo de plantas silvestres y cultivadas, (5) extracción de muestras in situ de carbón, madera, fragmentos óseos, conchas, coprolitos y restos de plantas para 


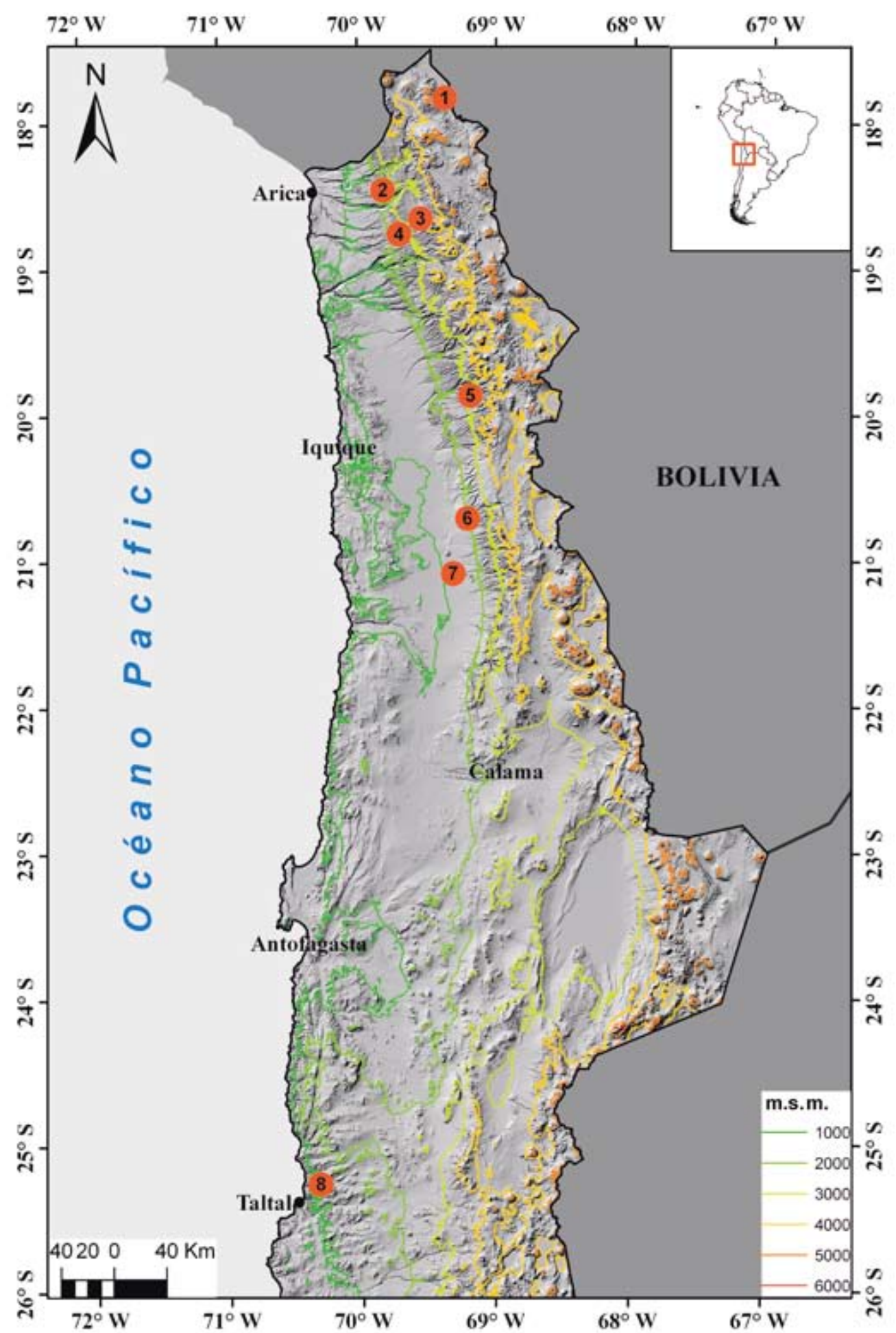

Figura 1. Principales sitios arqueológicos registrados por el proyecto en mapa con modelo de elevación (msm): (1) Hakenasa, (2) Alero Cardones, (3) Ipilla 2, (4) Pampa Timar, (5) Coscalla 3, (6) Chacarilla 15, (7) Quebrada Maní 12, (8) Quebrada Agua de Cascabeles.

Principal archaeological sites registered by our project, in a elevation model map (masl): (1) Hakenas, (2) Alero Cardones, (3) Ipilla 2, (4) Pampa Timar, (5) Coscalla 3, (6) Chacarilla 15, (7) Quebrada Maní 12, (8) Quebrada Agua de Cascabeles. 
datación por radiocarbono AMS, análisis de $\mathrm{ADN}^{1}$, antracológico ${ }^{2}$ e identificación de pigmentos ${ }^{3},(6)$ análisis tecnológicos de los conjuntos líticos, para determinar las cadenas operativas y los procesos técnicos y sociales implicados en la fabricación de los artefactos. Esto último con el objeto de reforzar el carácter temprano de estos yacimientos más allá de las características morfológicas de los instrumentos formatizados.

\section{Resultados}

Hasta la fecha se han identificado, entre los 1.000 a $3.000 \mathrm{msm}$ y desde los 18 hasta $\operatorname{los} 25^{\circ} \mathrm{S}$ (Valle de Lluta hasta Taltal), una serie de localidades con registros paleoambientales que muestran una mayor riqueza de flora y fauna durante la transición Pleistoceno-Holoceno con respecto a la actualidad. Estos resultados amplían y profundizan los antecedentes previos y constituyen una evidencia indirecta del aumento en la pluviosidad en la vertiente occidental de los Andes.

Se han colectado más de 100 paleomadrigueras en el área de estudio, donde destacan las localidades de Q. Ipilla, Pampa Timar, Q. Tarapacá, Sierra El Buitre, Q. Curiyuni y Pampa El Muerto. Un total de 32 paleomadrigueras y paleohumedales han sido datados con edades $\mathrm{C}^{14}$ cuyos rangos van entre el presente y los $>49,7 \mathrm{ka}$. Adicionalmente, se ha aplicado un método cuantitativo para estimar la precipitación media anual para una parte importante de este registro. Estos resultados, transversales a los propósitos del proyecto, han servido para afinar las secuencias paleoambientales.

Las prospecciones arqueológicas documentaron 14 localidades con ocupaciones humanas prehispánicas (Curiyuni, sector bajo del Valle de Lluta, sector intermedio de Quebrada Cardones, Quebrada La Higuera (Ipilla), Pampa Timar (al sur del poblado actual del mismo nombre), Coscalla, Alto Pachica, Pampa Quisma, Puquio Núñez, sectores bajo y medio de Quebrada de Chacarilla, sector bajo de Quebrada de Maní y sector medio de Quebrada Agua de Cascabeles). Se inventariaron 132 sitios arqueológicos, que en su conjunto confirmarían la existencia de condiciones de mayor humedad en el Desierto de Atacama durante distintos lapsos de tiempo, desde el Pleistoceno Tardío hasta el período Tardío. En el caso de las ocupaciones tempranas, los sitios consisten mayormente en talleres líticos sobre terrazas del Mioceno Tardío, ocupaciones efímeras en refugios rocosos y estructuras circulares en sitios abiertos.

A lo largo de este estudio, iniciado en el año 2007, se obtuvo un total de 57 fechas AMS para sitios arqueológicos (Ugalde et al. 2011), de las cuales 42 provienen de 14 yacimientos descubiertos y excavados por este proyecto, y 15 son muestras de sitios previamente intervenidos ${ }^{4}$ (Osorio et al. 2011). Cuatro de los sitios descubiertos por nosotros presentan instrumentos líticos, sobre la superficie, de tipología temprana. Sin embargo, sólo en dos de ellos las excavaciones arqueológicas produjeron material orgánico datable por $\mathrm{C}^{14}$ en asociación a otros restos de ocupación humana temprana. Primero, Ipilla 2 con un rango temporal que abarca desde el Holoceno Temprano hasta el Formativo y Quebrada Maní 12, con fechas correspondientes al Pleistoceno Tardío (Figura 1).

\section{Ipilla 2}

Está ubicado a $3.400 \mathrm{msm}\left(18,4^{\circ} \mathrm{S}\right)$ en la Quebrada La Higuera en la precordillera andina de Arica. Se halla junto a una paleovertiente registrada por Rech (2001), en cuyo perfil estratigráfico expuesto se fechó una capa de cenizas y desechos de talla lítica en 10,2 ka (Ipilla 1 de acuerdo a nuestra denominación, Quebrada La Higuera en la nomenclatura de Rech). Luego, como resultado de nuestras prospecciones, se encontró el sitio Ipilla 2. Este es un campamento abierto de aproximadamente $30.000 \mathrm{~m}^{2}$, en el que se obtuvieron 10 fechados por AMS, desde los estratos 5 a 13, de una excavación de $2 \mathrm{~m}^{2} \mathrm{y}$ una profundidad estratigráfica de $1,20 \mathrm{~m}$. Adicionalmente, se procesaron tres fechas AMS de un perfil expuesto en el borde exterior sur del sitio (Figura 2).

La ocupación se inicia a los 9,9 ka (estrato $12^{5}$ ) y se mantiene hasta los 9,6 ka (estrato 6). Entre estos estratos se intercalan cinco fechas que muestran un uso relativamente continuo del sitio (Tabla 1). Luego se verifica un hiato de 7.560 años, dado que el estrato 5 está datado en 2,05 ka. Es posible que esta discontinuidad temporal corresponda a una discordancia erosiva, ya que coincide con una transición abrupta de gravas fluviales a sedimentos finos con posterior desarrollo de suelos. Esto significa que una porción importante de sedimentos habrían sido erosionados desde el depósito estratigráfico original.

Rech (2001) señala que el paleohumedal de Ipilla tuvo varios eventos depositacionales, relacionados 


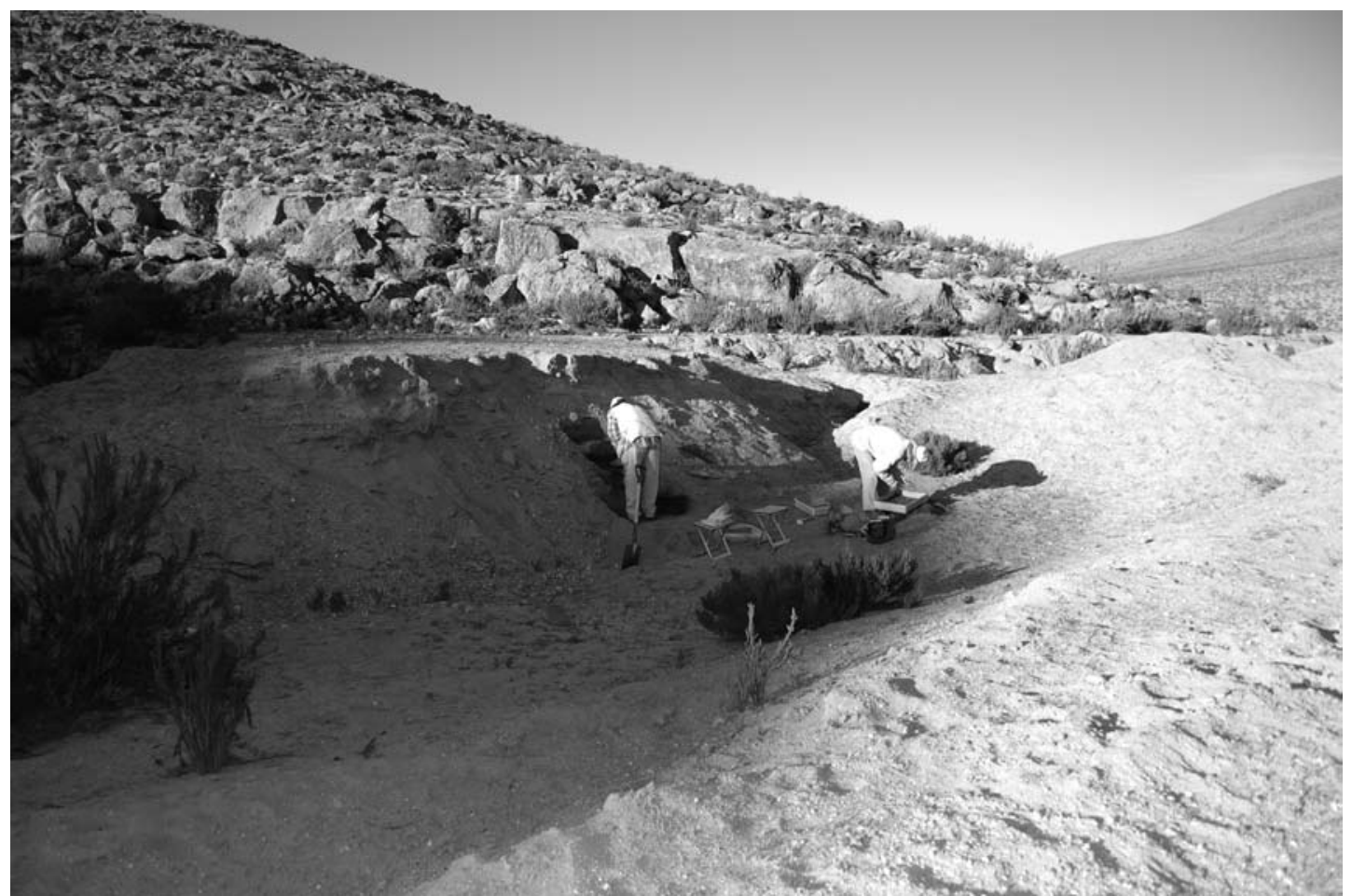

Figura 2. Perfil estratigráfico expuesto y ubicación del sitio Ipilla 2.

Stratigraphic profile and location of Ipilla 2 site.

con un aumento en el nivel de la napa freática a consecuencia de una mayor cantidad de precipitaciones en la precordillera. El primero (unidad B) ocurrió entre 12,25-9,49 ka, en clara concordancia con las fechas de la ocupación humana temprana ( 10 a 9,5 ka, ver Tabla 1). Rech, al igual que en la cronología de ocupación humana, no encontró eventos depositacionales entre 9,5 a 3,5 ka. Es más, las restantes unidades preservadas exhiben edades más recientes. La unidad C, por ejemplo, presenta fechas entre 3,47 a 3,29 ka (sin representación arqueológica). Mientras que la unidad más reciente (D2) muestra edades entre 1,13 a 0,4 ka, que podría coincidir con las ocupaciones con cerámica de los estratos 1 al 4, aún no fechados.

Análisis líticos en curso señalan una mayoría de desechos por sobre instrumentos formatizados para los niveles arcaico temprano, compuestos principalmente de dos materias primas silíceas que se repiten a lo largo de la secuencia. El conjunto se caracteriza por un gran número de desechos de retoque y de adelgazamiento bifacial, sin corteza, de tamaños pequeños $(\leq 20 \mathrm{~mm})$ y por la ausencia de núcleos. Lo anterior indica la presencia de las últimas etapas de la cadena operativa, es decir, la fabricación de instrumentos (Katherine Herrera, comunicación personal 20116). Análisis botánicos y zooarqueológicos están destinados a complementar los estudios líticos y a precisar el carácter y la función de estas ocupaciones. A pesar de las fechas tempranas este yacimiento no cumple del todo con las expectativas de nuestro modelo predictivo, dado que su data es posterior a los $11,5 \mathrm{ka}$ y se ubica a $3.400 \mathrm{~m}$, por encima de la cota máxima propuesta de $3.000 \mathrm{msm}$, en la transición entre el piso prepuñeno y puneño con un régimen climático de mayor precipitación ( 100 mm/año) que los pisos más desérticos de la precordillera andina.

\section{Quebrada Maní 12}

Se emplaza a $1.240 \mathrm{msm}\left(21^{\circ} \mathrm{S}\right)$ en el desagüe de la quebrada homónima, al suroeste de Guatacondo. Forma parte de un gran sistema de drenajes de la Pampa del Tamarugal, cuyas cabeceras se encuentran en la Sierra Moreno, ubicado en el núcleo hiperárido del Desierto de Atacama. Actualmente, las precipitaciones en la vecindad de los sitios arqueológicos son prácticamente cero (una tormenta por siglo quizás), situación que fue radicalmente diferente durante el 


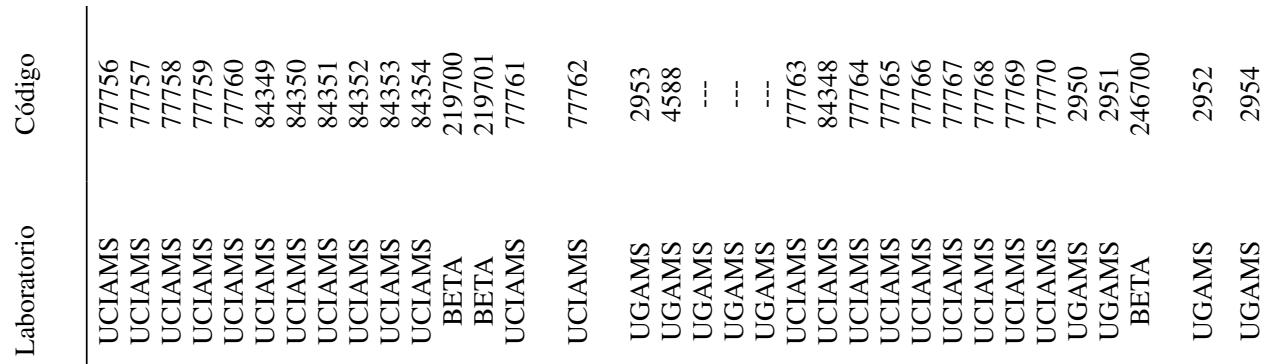

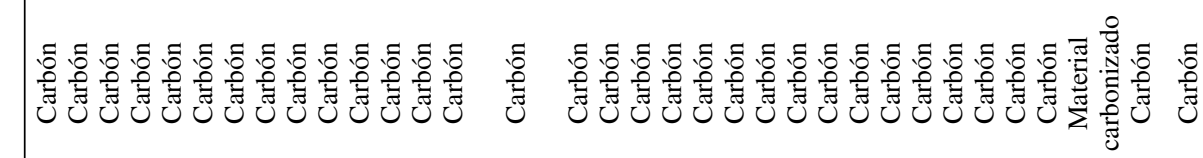

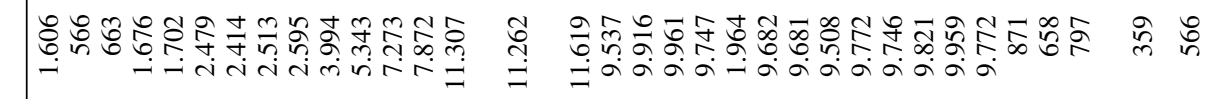
తే

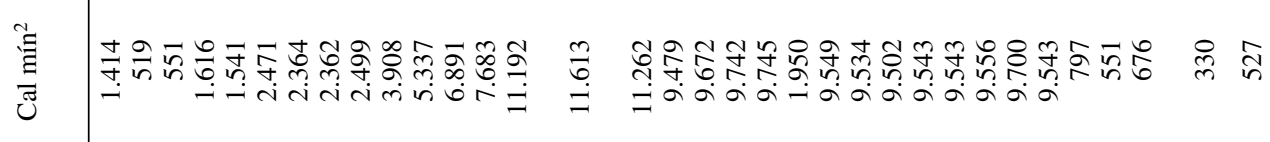

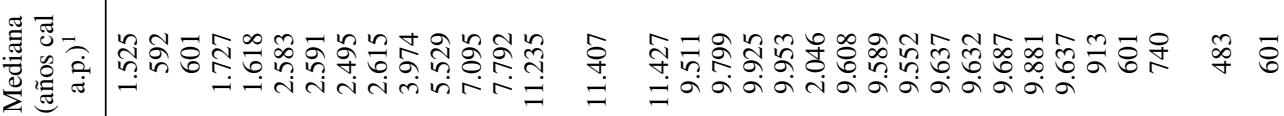

:

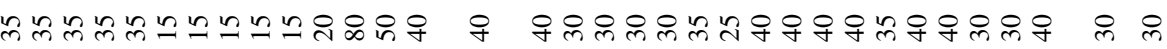

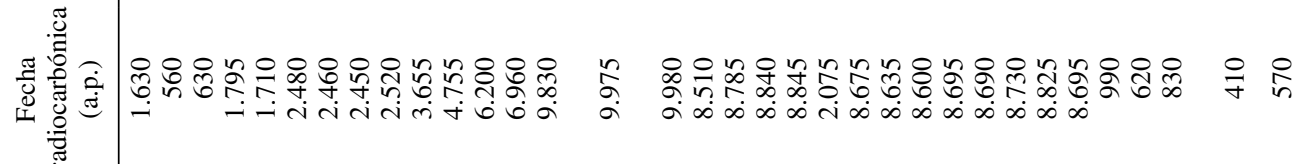
ฐ

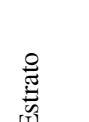

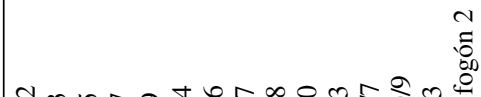
ข . : 离

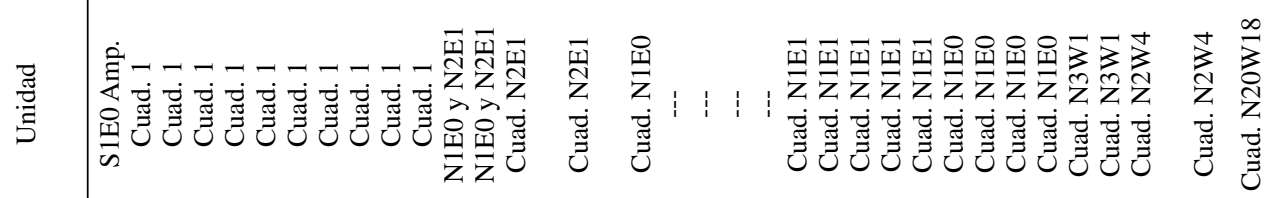

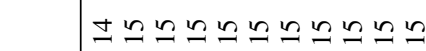




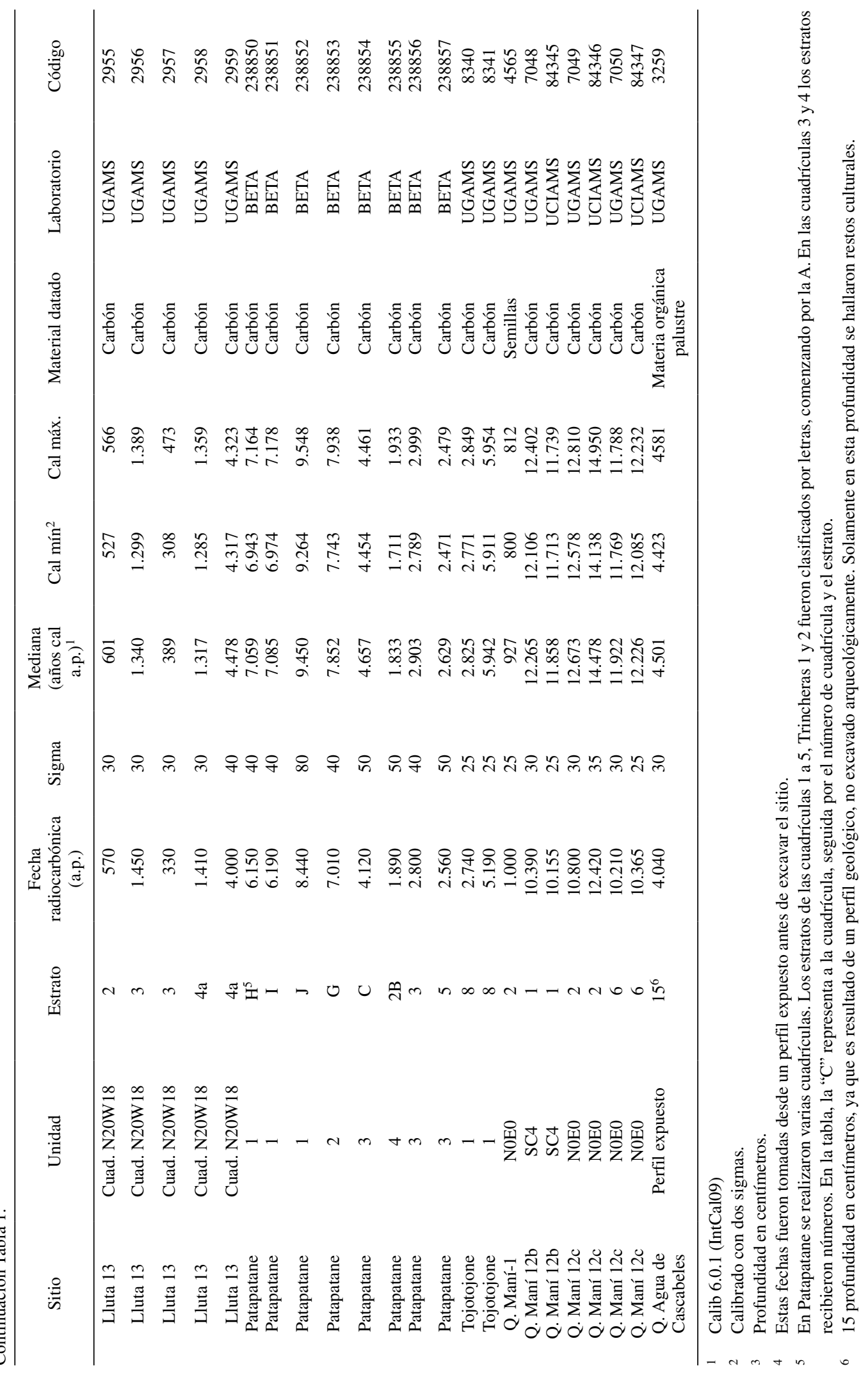


Pleistoceno Tardío (17-11 mil años atrás). Para ese entonces, mayores precipitaciones sobre la Sierra Moreno produjeron un incremento sostenido en la descarga de los ríos que bajaban, por lo que hoy son quebradas completamente secas y sin vegetación. En ellas se han registrado vestigios de bosques en galería con vegetación (i.e. sauce nativo o k'ero, Escallonia angustifolia) que actualmente existe sólo en quebradas con flujo perenne, como la de Camiña (Nester et al. 2007).

Parajes como el de Maní poblados por vegetación fueron verdaderos imanes para la fauna y fuente constante de agua y recursos vegetales durante el Pleistoceno Tardío, por lo que pudieron constituir lugares ideales para pequeñas bandas de cazadores-recolectores. En esta zona de quebrada se han documentado y fechado evidencias de madera fósil, que indicarían un ambiente con mayor disponibilidad de agua en superficie alrededor de los 16,4 a 13,7 ka, periodo sincrónico con el paleolago Tauca en el altiplano boliviano (Nester et al. 2007). Lo interesante es que el agua de Quebrada Maní no provino del incremento de lluvias sobre el desierto (que permanecía árido y sin precipitaciones constantes), sino de cursos de aguas perennes producto del incremento de lluvias en la cordillera hacia el este (Gayó et al. 2009; Nester et al. 2007).

El sitio Maní 12 ocupa mayormente la fracción noreste de un remanente erosivo de una terraza aluvial del Mioceno Tardío (Figura 3), con una extensión de $\sim 1.6 \mathrm{~km}^{2}$. Accedimos a este sector en virtud de los antecedentes paleoambientales reseñados. El sitio se compone superficialmente de múltiples eventos de talla de materias primas locales (i.e. arenisca cuarcífera) y foráneas (silíceas, de buena calidad, cuya cantera se ubica a unos $20 \mathrm{~km}$ al norte de QM12, Nicolás Blanco comunicación personal 2011). Exhibe principalmente lascas, desechos de talla bifacial y bifaces en distintas etapas de manufactura. Entre estos últimos destacan varias puntas de proyectil características del Arcaico Temprano, como las puntas Patapatane, y una punta que recuerda el patrón Tuina (Figura 4). Adicionalmente, se registran algunos fragmentos de artefactos de molienda sobre materias primas locales, caracterizados por una superficie relativamente cóncava

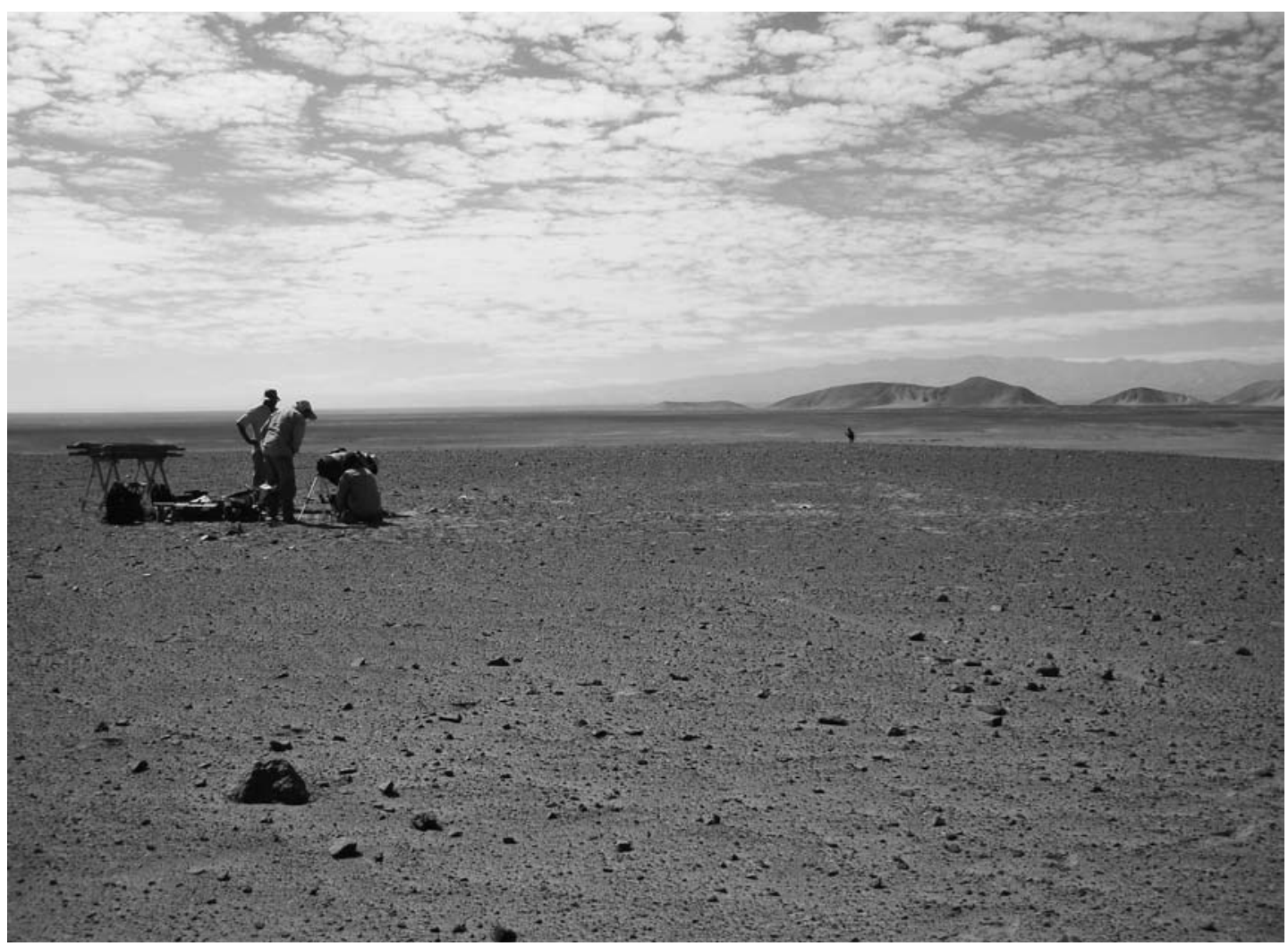

Figura 3. Vista del sitio Q. Maní 12c, sobre el remanente erosivo de terraza miocénica. View of site $\mathrm{Q}$. Maní $12 c$ upon the Miocenic erosive remnant terrace. 


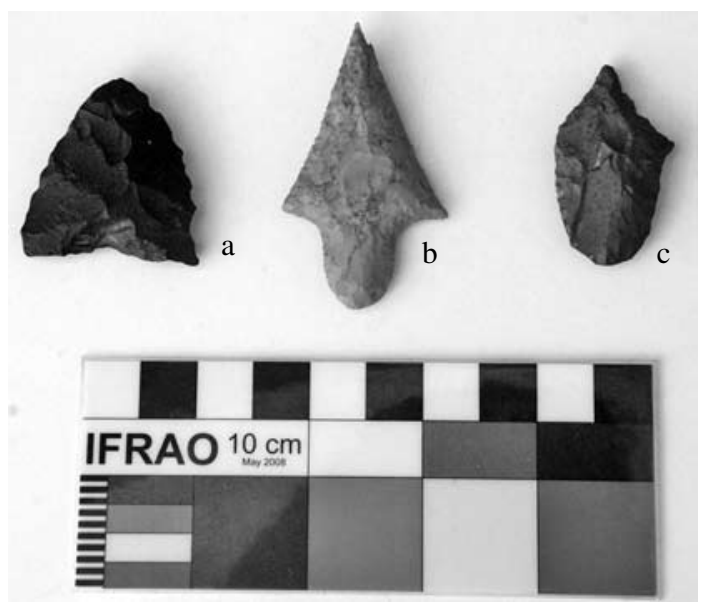

Figura 4. Puntas de proyectil de la superficie de QM12: (a) Triangular tipo "Tuina"; (b) Triangular con pedúnculo y hombros prominentes, similar a un tipo del sitio "Las Cuevas"; (c) Punta Patapatane retomada.

Surface projectile points from QM12: (a) Tuina-like triangular point; (b) Stemmed triangular with prominent shoulders, similar to one of "Las Cuevas" types; (c) Patapatane reworked point.

y bordes pulidos. Asociados a algunos eventos de talla, destaca la presencia de minúsculas astillas de hueso de fauna mayor, que fue el criterio para elegir los lugares de excavación, identificados como QM12a, QM12b y QM12c.

En QM12c, un área de $2 \times 2 \mathrm{~m}$ y $30 \mathrm{~cm}$ de profundidad, reveló un conjunto de rasgos que incluyen: un fogón en cubeta, concentraciones de carbón y cenizas, dos postes y astillas de madera, pigmentos de color rojo, conchas del Pacífico, fragmentos óseos de fauna como camélidos y coprolitos de animales. El conjunto lítico incluye desechos similares a aquellos de superficie (muchos de ellos fragmentados), muy pocos fragmentos de instrumentos y escasos fragmentos de posibles núcleos. Cuatro fechas de AMS sobre carbones recolectados in situ desde dos concentraciones de carbón fueron procesadas en los laboratorios UCIAMS y UGAMS. Las muestras de la primera concentración de carbones, ubicadas en el estrato 2, arrojaron fechas de 14,48 y 12,67 ka, respectivamente. Los resultados del segundo evento, del estrato 4, presentan dataciones más cercanas entre sí de 11,92 y 12,23 ka respectivamente (Tabla 1). Confirma la data temprana de QM12c la total ausencia de elementos tardíos, como cerámica, vegetales cultivados, metales, entre otros.

Estas fechas muestran una inversión estratigráfica que pudo ser la consecuencia de excavaciones practicadas por sucesivos ocupantes del sitio, para acomodar postes y fogones, entre otras actividades. Luego, es importante señalar que tres de las fechas son consistentes entre sí, al ubicarse entre 12,67 y $11,92 \mathrm{ka}$, lo que contrasta con la fecha de 14,48 ka, que podría corresponder a carbones de maderas más antiguas que se recogieron para combustionarlas.

QM12b, a unos $40 \mathrm{~m}$ de QM12c, corresponde a un sector de mayor densidad de restos líticos sobre la superficie. Las excavaciones de este sector comenzaron con una calicata de $70 \times 70 \mathrm{~cm}$, ubicada en el extremo suroeste de QM12b, y no arrojaron materiales para datación. Adicionalmente, se definió un área de $5 \times 10 \mathrm{~m}$, separada en unidades de 2,5x2,5 m, para recolectar todos los artefactos de superficie (ver metodología). Además, se raspó una capa superficial de 1 a $2 \mathrm{~cm}$ para complementar la recolección superficial. A consecuencia de ello, en una de las unidades afloró una concentración de cenizas y algunos carbones desde $\sim 2$ a $5 \mathrm{~cm}$ de profundidad. Como en el caso de QM12c, en el laboratorio UGAMS se obtuvo un resultado de 12,27 ka, mientras que del laboratorio UCIAMS se obtuvo un resultado 11,86 ka (Tabla 1). Por último, en el sector QM12a se realizó una calicata de 50x50 $\mathrm{cm}$, donde se obtuvieron escasos materiales culturales y ningún elemento para datación.

En toda la extensión del sitio Quebrada Maní 12 se registran escasos elementos tardíos en superficie (dos fragmentos cerámicos a $85 \mathrm{~m}$ de QM12c). Esto contrasta con la densidad y amplitud de la ocupación tardía emplazada en terrazas fluviales formadas durante el Pleistoceno Tardío, que se ubican en un nivel más bajo respecto de QM12. Allí se encuentran terrazas de cultivo, canales de regadío, estanques para almacenar agua, rocas con grabados rupestres, estructuras habitacionales, hornos para cerámica, postes de madera, restos líticos y cerámica. Estos sitios han sido catastrados como QM2 a QM11 y QM13.

De un total de 13 sitios registrados, sólo Quebrada Maní 12 presenta evidencias tempranas. En otro remanente erosivo de una terraza del Mioceno Tardío se ubica el sitio Quebrada Maní 1 (QM1), donde se realizaron tres calicatas, obteniéndose una fecha AMS correspondiente al período Intermedio Tardío (0,9 ka, Tabla 1). Este sitio presenta una menor densidad de artefactos líticos superficiales en relación a QM12 y no se observaron instrumentos diagnósticos tempranos. QM1 presenta geoglifos en forma de emplantillados circulares de piedra (identificados por Luis Briones, comunicación 
personal, 2010) y muy escasa presencia de cerámica. Además, al norte de QM12 se exploró otro remanente miocénico registrado como QM13, donde sólo se identificaron elementos tardíos (pircas, geoglifos y desechos de talla lítica).

\section{Discusión y Conclusiones}

Los resultados de nuestros estudios paleoambientales demuestran que el Desierto de Atacama tuvo condiciones más favorables para la habitación humana que en la actualidad, desde el Pleistoceno Tardío hasta el Holoceno Temprano (17 a 9,5 ka). Esta situación se dio particularmente en ciertas localidades de la franja hiperárida entre $1.000 \mathrm{y}$ $3.000 \mathrm{msm}$, por efecto de un alza de las precipitaciones en la precordillera. Quebrada Maní 12 corrobora el modelo planteado, vale decir: (1) estas condiciones generaron un ambiente propicio para la ocupación humana en un área que es hoy completamente árida y que no había sido explorada para la identificación de sitios arqueológicos tempranos, y (2) esta localidad ribereña fue ocupada efectivamente por grupos cazadores-recolectores que aprovecharon un paleohábitat integrado por un estero, arboledas y la presencia de animales como camélidos. Estas condiciones paleoambientales estuvieron vigentes hasta los $\sim 11 \mathrm{ka}$.

Quebrada Maní 12 es el único sitio arqueológico para el Atacama Norte con cinco fechas AMS que fluctúan entre $\sim 11,9$ y 12,7 ka. Se agrega una fecha de 14,48 ka, considerada anómala, puesto que se desvía significativamente del promedio de las otras cinco. Estimamos que esta datación proviene de carbones de madera expuesta por cerca de 2.000 años antes de la ocupación humana, puesto que evidencias de Escallonia y otras especies arbustivas y arbóreas se registran en Quebrada Maní a partir de $\sim 17 \mathrm{ka}$ (Nester et al. 2007).

QM12 está ubicado en uno de los remanentes erosivos de terrazas relictas del Mioceno, con poca actividad en época holocénica (escasa erosión fluvial y depositación eólica). Adicionalmente, la hiperaridez permitió una excelente conservación de los materiales en QM12c, donde se cuenta con evidencias poco comunes para sitios de data temprana, como postes de madera, pigmentos, restos óseos y coprolitos. Estimamos que este no sería un caso aislado, en la medida que existen otras localidades con condiciones paleoecológicas similares (paleovertientes y paleobosques) que aún no han sido prospectadas y menos excavadas. Por ejemplo, en la localidad de Puquio Núñez, ubicada al sureste de Matilla en la región de Tarapacá, se registró un sitio sobre cuya superficie no existe prácticamente ningún material arqueológico tardío o evidencias históricas, sino solamente núcleos y desechos líticos, acompañados de algún instrumento tosco y poco formatizado (Ugalde 2009). En contraste, el área alrededor de la vertiente de agua contigua a este sitio, y ubicada en un plano más bajo, muestra evidencias de que fue ocupada desde tiempos prehispánicos tardíos hasta época reciente (Lautaro Núñez, comunicación personal 2007).

Por otra parte, varias localidades con registros paleoambientales que indican igualmente una mayor humedad para la época en estudio, como Lomas de Sal y Mamiña, no presentan evidencias arqueológicas tempranas hasta ahora. Cuatro fechas del Alero Cardones arrojaron resultados para el período Intermedio Tardío, mientras que los radiocarbonos de Quebrada Agua de Cascabeles e Ipilla son de época arcaica. En Pampa Timar, en tres calicatas excavadas, simplemente no encontramos materiales susceptibles de ser datados.

Si bien Maní 12 es el único sitio temprano registrado por el proyecto, al sumarse a los yacimientos ya conocidos de fechas sincrónicas para el Desierto de Atacama Central y Sur (Tuina-1, Tuina-5, San Lorenzo, Tulán 109, Salar de Punta Negra-1, Alero el Pescador y San Ramón 15), viene a confirmar la antigüedad y diversidad cultural de los primeros poblamientos humanos de Sudamérica (de Souza 2004; Dillehay 2000, 2002; Grosjean et al. 2005b; Núñez et al. 2002; Osorio et al. 2011; Salazar et al. 2011). Cabe destacar, por ejemplo, la ausencia de evidencias de megafauna extinta y puntas "cola de pescado" en la mayoría de estos sitios (Jackson et al. 2004).

Todos estos yacimientos, sin embargo, son antecedidos en 2.000 años por Monte Verde (Dillehay 1997), por lo que, dada la presencia de condiciones paleoecológicas propicias desde los $17 \mathrm{ka}$ en la cuenca de Maní y en otras localidades del desierto de Atacama, se podría esperar la existencia de sitios anteriores $13 \mathrm{ka}$.

Dados estos antecedentes, postulamos que el Desierto de Atacama pudo ser una ruta de circulación para los primeros pobladores de Sudamérica, desde la costa sur peruana hacia Chile Central (Santa Julia, Quereo, Tagua Tagua) y Centro-Sur (Monte Verde). Por otra parte, la presencia de 
sitios tempranos en la costa sur peruana, conchas del Pacífico en el sitio QM12c y algas marinas en Monte Verde sustenta la hipótesis de una vía migratoria a lo largo de la costa del Pacífico desde el Istmo de Panamá (Dillehay et al. 2008; Jackson et al. 2007; Montané 1968; Núñez, Varela, Casamiquela, Schiappacasse, et al. 1994; Núñez, Varela, Casamiquela y Villagrán 1994; Richardson 1983, 2006; Rothhammer y Dillehay 2009; Sandweiss et al. 1998).

Los estudios arqueológicos en Quebrada Maní verifican que los grupos humanos se establecieron en el lugar mientras existieron recursos bióticos. Después de los primeros poblamientos (13.000-11.000 años cal. a.p.) no hubo ocupaciones humanas hasta los períodos Medio (ca. 1.650-950 a.p.) e Intermedio Tardío (ca. 950-550 a.p.), cuando existió un leve aumento de la napa freática y un mejoramiento de las condiciones paleoambientales (Gayó et al. 2010; Nester et al. 2007). Los asentamientos tardíos no se ubicaron en los remanentes de terrazas miocénicas, lo cual asegura poca perturbación postocupacional en los sitios tempranos. Las alteraciones observadas en QM12c (inversión cronológica, fragmentación de líticos) corresponderían a la época de ocupación temprana, que se habría caracterizado por sucesivas visitas de corta duración al sitio. En suma, localidades como Maní son ideales para el estudio del poblamiento temprano de Sudamérica.

Agradecimientos: Proyecto Fondecyt 1070140 (para CMS, CL y CS), Universidad de Tarapacá, Instituto de Alta Investigación y Centro de Investigaciones del Hombre en el Desierto (CIHDE). Agradecemos la participación activa de Luis Briones, Alexandre Chevalier, Francisca Díaz, Soledad Fernández, Anita Flores, Matías Frugone, Katherine Herrera, Sussy Rodríguez, Delphine Joly, José Müller, Isabel Mujica, Félix Olivares, Rosario Palomo, Maritza Ramos, Carolina Santoro, David Sepúlveda, Marcela Sepúlveda, Anita Valentin, Daniela Valenzuela, Leslia Véliz, Andrés Vilca, Natalia Villavicencio, Paola Villegas. Estudiantes de $3^{\text {er }}$ año de Arqueología y Antropología de la Universidad de Tarapacá. A su vez, agradecemos el apoyo del Proyecto Fondecyt 1100916 (a CL y CS), los proyectos PFB-23 y P05-002 para el IEB, y FONDAP 1501-2001 para CASEB. Finalmente agradecemos a los editores Mark Aldenderfer y Luis Angel Flores y a los revisores anónimos.

\section{Referencias Citadas}

Anderson, D.G. y J.C. Gillam 2000. Paleoindian colonization of the Americas: implications from an examination of physiography, demography, and artefact distribution. American Antiquity 65:43-66:43-66.

Betancourt, J.L., C. Latorre, J.A. Rech, J. Quade y K.A. Rylander 2000. A 22,000-yr record of monsoonal precipitation from Northern Chile's Atacama Desert. Science 289:1542-1546.

Betancourt, J.L. y B. Saavedra 2002. Nuevo método paleoecológico para el estudio de zonas áridas en Sudamérica: paleomadrigueras de roedores. Revista Chilena de Historia Natural 75:527-546.

Betancourt, J.L., T.R. Van Devender y P.S. Martin 1990. Packrat Middens: The Last 40,000 Years of Biotic Change. University of Arizona Press, Tucson.

Dauelsberg, P. 1983. Tojo-Tojone: un paradero de cazadores arcaicos (características y secuencias). Chungara 11:11-30.

de Souza, P. 2004. Cazadores recolectores del Arcaico Temprano y Medio en la cuenca superior del río Loa: sitios, conjuntos líticos y sistemas de asentamiento. Estudios Atacameños 27:7-43.

deFrance, S.D. y A. Umire 2004. Quebrada Tacahuay: un sitio marítimo del Pleistoceno tardío en la costa sur del Perú. Chungara Revista de Antropología Chilena 36:257-278.

Dillehay, T.D. 1989. Monte Verde: A Late Pleistocene Settlement in Chile. Volume 1. Smithsonian Institution, Washington.
- - - 1997. Monte Verde: A Late Pleistocene Settlement in Chile. Volume 2. Smithsonian Institution, Washington.

- - - 2000. The Settlement of the Americas: A New Prehistory. Basic Books, New York.

_. - 2002. Climate and human migrations. Science 298:764765.

Dillehay, T.D., C. Ramírez, M. Pino, M.B. Collins, J. Rossen y J.D. Pino-Navarro 2008. Monte Verde: seaweed, food, medicine, and the peopling of South America. Science 320:784-786.

Gayó, E.M., C. Latorre, T.E. Jordan y P.L. Nester 2009. Fantasmas de bosques y agua fósil en la Pampa del Tamarugal, norte de Chile. En Resúmenes extendidos del XII Congreso Geológico Chileno, Santiago.

Gayó, E.M., C. Latorre y C.M. Santoro 2010. Centennial-scale climate changes during the last 2,000 years in the Atacama Desert $\left(16^{\circ}-26^{\circ} \mathrm{S}\right)$. Ponencia presentada en $A G U$ Meeting of the Americas, Foz do Iguaçu, Brasil.

Grosjean, M., L. Núñez y I. Cartajena 2005a. Cultural response to climate change in the Atacama Desert. En $23^{\circ}$ South: Archaeology and Environmental History of the Southern Deserts, editado por M. Smith y P. Hesse, pp. 156-171. National Museum of Australia Press, Canberra.

_ _ - 2005b. Paleoindian occupation of the Atacama Desert, northern Chile. Journal of Quaternary Science 20:643-653. 
Holmgren, C.A., J.L. Betancourt, K.A. Rylander, J. Roque, O. Tovar, H. Zeballos, E. Linares y J. Quade 2001. Holocene vegetation history from fossil rodent middens near Arequipa, Peru. Quaternary Research 56:242-251.

Holmgren, C.A., E. Rosello, C. Latorre y J.L. Betancourt 2008. Late-Holocene fossil rodent middens from the Arica region of northernmost Chile. Journal of Arid Environments 72:677-686.

Jackson, D., C. Méndez y P. de Souza 2004. Poblamiento paleoindio en el norte-centro de Chile: evidencias, problemas y perspectivas de estudio. Complutum 15:165-176.

Jackson, D., C. Méndez, R. Seguel, A. Maldonado y G. Vargas 2007. Initial occupation of the Pacific coast of Chile during late Pleistocene times. Current Anthropology 48:725-731.

Keefer, D., S. deFrance, M. Moseley, J. Richardson III, D. Satterlee y A. Day-Lewis 1998. Early maritime economy and El Niño events at Quebrada Tacahuay. Science 281:1833-1835.

Kelly, R. 2003. Maybe we do know when people first came to North America; and what does it mean if we do? Quaternary International 109-110:133-145.

Lanning, E.P. 1967. Early man in South America. Scientific American 217:44-50.

- . - 1973. Burin industries in the Pleistocene of the Andes. Estudios Atacameños 1:21-37.

Latorre, C., J.L. Betancourt y M.T.K. Arroyo 2006. Late Quaternary vegetation and climate history of a perennial river canyon in the Río Salado basin $\left(22^{\circ} \mathrm{S}\right)$ of northern Chile. Quaternary Research 65:450-466.

Latorre, C., J.L. Betancourt, J.A. Rech, J. Quade, C. Holmgren, C. Placzek, A. Maldonado, M. Vuille y K.A. Rylander 2005. Late Quaternary history of the Atacama Desert. En $23^{\circ} \mathrm{S}$ : The Archaeology and Environmental History of the Southern Deserts, editado por M. Smith y P. Hesse, pp. 73-90. National Museum of Australia Press, Canberra.

Latorre, C., J.L. Betancourt, K.A. Rylander y J. Quade 2002. Vegetation invasions into absolute desert: A 45,000-yr rodent midden record from the Calama-Salar de Atacama basins, Northern Chile $\left(22-24^{\circ} \mathrm{S}\right)$. Geological Society of America Bulletin 114:349-366.

Latorre, C., J.L. Betancourt, K.A. Rylander, J. Quade y O. Matthei 2003. A Vegetation history from the arid prepuna of northern Chile $\left(22-23^{\circ} \mathrm{S}\right)$ over the last 13,500 years. Palaeogeography, Palaeoclimatology, Palaeoecology 194:223-246.

Le Paige, G. 1965. San Pedro de Atacama y sus zonas. Anales de la Universidad del Norte 4:1-99.

- - - 1970. Industrias Líticas de San Pedro de Atacama. Coedición Universidad del Norte-Orbe, Santiago.

Lynch, T.F. 1980. Presencia y adaptación post-glacial del hombre en los Andes sudamericanos. Chungara 6:96-123.

- - - 1986. Un reconocimiento arqueológico en el salar de Punta Negra, II Región. Chungara 16/17:75-88.

Montané, J. 1968. Paleo-indian remains from Laguna Taguatagua, Central Chile. Science 161:1137-1138.
Moreno, A., S. Giralt, B. Valero-Garcés, A. Sáez, R. Bao, R. Prego, J.J. Pueyo, P. González-Sampériz y C. Taberner 2007. A $14 \mathrm{kyr}$ record of the tropical Andes: The Lago Chungará sequence $\left(18^{\circ} \mathrm{S}\right.$, Northern Chilean Altiplano). Quaternary International 161:4-21.

Moreno, A., C.M. Santoro y C. Latorre 2009. Climate change and human occupation in the northernmost Chilean Altiplano over the last $\sim 11,500 \mathrm{cal}$ yr BP. Journal of Quaternary Science 24:373-382.

Nester, P., E. Gayó, C. Latorre, T.E. Jordan y N. Blanco 2007. Perennial stream discharge in the hyperarid Atacama Desert of northern Chile during the latest Pleistocene. Proceedings of the National Academy of Sciences 104:19724-19729.

Núñez, L. 1975. Programa Paleo-Indio en Chile. Estudios Atacameños 3:89-93.

Núñez, L., M. Grosjean e I. Cartajena 2002. Human occupations and climate change in the Puna de Atacama, Chile. Science 298:821-824.

- - - 2005. Ocupaciones Humanas y Paleoambientes en la Puna de Atacama. Instituto de Investigaciones y Museo, Universidad Católica del Norte, Taraxacum, San Pedro de Atacama.

Núñez, L., J. Varela, R. Casamiquela, V. Schiappacasse, H. Niemeyer y C. Villagrán 1994. Cuenca de Taguatagua en Chile: el ambiente del Pleistoceno superior y ocupaciones humanas. Revista Chilena de Historia Natural 67:503-519.

Núñez, L., J. Varela, R. Casamiquela y C. Villagrán 1994. Reconstrucción multidisciplinaria de la ocupación prehistórica de Quereo, centro de Chile. Latin American Antiquity 5:99-118.

Osorio, D., D. Jackson, P. Ugalde, C. Latorre, R. de Pol-Holz y C.M. Santoro 2011. The Hakenasa cave and its relevance for the peopling of the southern Andean Altiplano. Antiquity 85 , en prensa.

Placzek, C., J. Quade, J.L. Betancourt, P.J. Patchett, J.A. Rech, C. Latorre, A. Matmon, C. Holmgren y N.B. English 2009. Climate in the dry central Andes over geologic, millennial, and interannual timescales. Annals of the Missouri Botanical Garden 96:386-397.

Quade, J., J.A. Rech, J.L. Betancourt, C. Latorre, B. Quade, K.A. Rylander y T. Fisher 2008. Paleowetlands and regional climate change in the central Atacama Desert, northern Chile. Quaternary Research 69:343-360.

Rech, J. 2001. Late Quaternary Paleohydrology and Superficial Processes of the Atacama Desert, Chile: Evidence from Wet land Deposits and Stable Isotopes of Soil Salts. Ph.D. Dissertation, Department of Geosciences, University of Arizona, Tucson.

Rech, J., J. Quade y J.L. Betancourt 2001. Paleoclimatic reconstruction of the Atacama Desert (18-26 $\left.6^{\circ}\right)$ : evidence from wetland deposits. Proceedings from the Central Andean Paleoclimate Workshop, Tucson, Arizona.

- - - 2002. Late Quaternary paleohydrology of the Central Atacama Desert $\left(22-24^{\circ}\right)$, Chile. Geological Society of America Bulletin 114:334-348.

Richardson, J.B. III. 1983. The Chira beach ridges, sea level change and the origins of maritime economies on the Peruvian coast. Annals of Carnegie Museum 52:265-276. 
- - - 2006. Looking in the right places: Maritime adaptations in northeastern North America and the Central Andes. En From the Arctic to Avalon: Papers in Honor of Jim Tuck, editado por L. Rankin y P. Ramsden, pp. 83-98. BAR International Series 1507.

Rothhammer, F. y T.D. Dillehay 2009. The Late Pleistocene colonization of South America: an interdisciplinary perspective. Annals of Human Genetics 73:540-549.

Salazar, D., D. Jackson, J.L. Guendon, H. Salinas, D. Morata, V. Figueroa, G. Manríquez y V. Castro 2011. Early evidence (ca. 12,000 BP) for iron oxide mining on the Pacific coast of South America. Current Anthropology 52:463-475.

Sandweiss, D., H. McInnis, R. Burger, A. Cano, B. Ojeda, R. Paredes, M. Sandweiss y M. Glascock 1998. Quebrada Jaguay: Early south american maritime adaptations. Science 281:1830-1832.

Santoro, C.M. y C. Latorre 2009. Propuesta metodológica interdisciplinaria para poblamientos humanos Pleistoceno tardío/Holoceno temprano, precordillera de Arica, Desierto de Atacama Norte. Andes 7:11-35.
Schiappacasse, V. y H. Niemeyer 1984. Descripción y Análisis Interpretativo de un Sitio Arcaico Temprano en la Quebrada de Camarones. Museo Nacional de Historia Natural. Publicación Ocasional 41. Santiago.

Ugalde, P.C. 2009. Evaluación de Meteorizaciones Diferenciales en Instrumentos Líticos de Sitios Superficiales del Área Quebrada de Chacarilla, Región de Tarapacá, Chile. Memoria para optar al título de Arqueóloga, Facultad de Ciencias Sociales, Universidad de Chile, Santiago.

Ugalde, P.C., C. Salas, C. Latorre, D. Osorio, D. Jackson y C.M. Santoro 2011. Poblamiento temprano del norte de Chile $\left(18-25^{\circ} \mathrm{S}\right)$ : estudio interdisciplinario arqueológico y paleoambiental. Enviado a Actas del XVIII Congreso de Arqueología Chilena. Valparaíso.

Williams, A., C.M. Santoro, M.A. Smith y C. Latorre 2008. The impact of ENSO in the Atacama Desert and Australian arid zone: Exploratory time-series analysis of archaeological records. Chungara Revista de Antropología Chilena 40:245-259.

\section{Notas}

1 Australian Centre for Ancient DNA, a través de Alan Cooper.

2 UMR 6566, CREAAH, Archéosciences-Rennes, Université de Rennes, a través de Delphine Joly.

3 C2RMF, Centre de Recherche et de Restauration des Musées de France, a través de Marcela Sepúlveda.
4 Materiales conservados en el Museo Arqueológico Universidad de Tarapacá, San Miguel de Azapa, Arica.

5 El estrato 13 tiene una fecha de 9.637 cal a.p., algo más reciente que la del estrato 12 .

6 Memoria para optar al título de Arqueóloga, Universidad de Tarapacá, en proceso. 